PROCEEDINGS OF THE

AMERICAN MATHEMATICAL SOCIETY

Volume 137, Number 4, April 2009, Pages 1347-1356

S 0002-9939(08)09667-6

Article electronically published on October 6, 2008

\title{
LINEARLY APPROXIMATABLE FUNCTIONS
}

\author{
THIERRY DE PAUW AND AMOS KOELLER
}

(Communicated by Tatiana Toro)

\begin{abstract}
The notion of uniform linear approximatability generalizes that of being continuously differentiable. It occurs, e.g., in viscosity solutions of some degenerate partial differential equations. We establish the Hölder continuity of uniformly linearly approximatable functions, and we show that functions which are nowhere linearly approximatable form a residual collection of the appropriate Hölder space. Finally, we prove an analog of the implicit function theorem applied to level sets.
\end{abstract}

\section{INTRODUCTION}

We say that $u: \mathbb{R}^{m} \rightarrow \mathbb{R}$ is linearly $\varepsilon$-approximatable at $x \in \mathbb{R}^{m}$ at scale $r>0$ if there exists a vector $e \in \mathbb{R}^{m}$ such that

$$
|u(x+h)-u(x)-\langle e, h\rangle| \leqslant \varepsilon r
$$

whenever $h \in B(0, r)$. For instance, if $u$ is differentiable at $x$ one can take $e=$ $\nabla u(x)$ and $r>0$ small enough depending upon $\varepsilon>0$. Points where a given Lipschitzian $u$ is linearly $\varepsilon$-approximatable occur in a set whose complement is small in a sense more restricted than being negligible with respect to the Lebesgue measure, thus providing a version of Rademacher's Theorem; see e.g. [5] (here $m \geqslant 2$ ). Other instances of linearly $\varepsilon$-approximatable functions have occurred recently as $\infty$-harmonic functions; see [3, 10] and [1] for a survey. These are viscosity solutions of the equation $\left\langle\nabla|\nabla u|^{2}, \nabla u\right\rangle=0$.

In general, though, the existence of an $\varepsilon$-gradient $e=e(\varepsilon)$ at scale $r=r(\varepsilon)$ (at a fixed point $x \in \mathbb{R}^{m}$ ) as in (1) does not imply the convergence of $e(\varepsilon)$ to a limit as $\varepsilon \rightarrow 0$; see Example 2.2. Despite this potential unsettling behavior of $\varepsilon$ gradients, the linear $\varepsilon$-approximatability of $u$ at every point $x \in S$ of some compact set $S \subset \mathbb{R}^{m}$ and for every uniformly small scale $0<r \leqslant r_{0}$ implies the continuity of $u$ restricted to $S$ together with an estimate of its oscillation. In fact we show in Theorem 2.7 that for every $0<\alpha<1$ there exists $C=C\left(u, S, \varepsilon, r_{0}, \alpha\right)$ such that

$$
|u(y)-u(x)| \leqslant C|y-x|^{\alpha}
$$

whenever $x, y \in S$. This conclusion is reached regardless of the value of $\varepsilon>0$.

Next we show that within the space of functions Hölder continuous with respect to each exponent $0<\alpha<1$ the collection of those which nowhere are linearly

Received by the editors April 10, 2008.

2000 Mathematics Subject Classification. Primary 26B35, 26B10, 46E10.

The first author is a chercheur qualifié of the Fonds National de la Recherche Scientifique, Belgium. 
approximatable is residual, thus generalizing a classical result due independently to S. Banach [2] and S. Mazurkiewicz [7. Theorem 3.3] is stronger than its classical analog because linear approximatability is weaker than differentiability, and because the relevant topology turning the collection of Hölder continuous functions into a complete metric space involves their oscillation.

The notion of linearly $\varepsilon$-approximatability of $u$ on uniformly small scales can be regarded as a generalization of the concept of being continuously differentiable. Indeed the latter implies a Lipschitz estimate for $u$ whereas, as we have seen, the former implies Hölder estimates for $u$. A consequence of this is a weak analog of the implicit function theorem. In case $u$ is continuously differentiable at $x$ and $\nabla u(x) \neq 0$, the level set $\{y: u(y)=u(x)\}$ is, in a neighborhood of $x$, a submanifold of dimension $m-1$. In case $u$ is linearly $\varepsilon$-approximatable on uniformly small scales and there are $\varepsilon$-gradients with length uniformly bounded away from zero, the level set $\{y: u(y)=u(x)\}$ is, in a neighborhood of $x$, Hölder homeomorphic to an $(m-1)$-dimensional ball; see Theorem 4.1. We obtain this result as a corollary of E.R. Reifenberg's topological disk Theorem, [9], whence the need for $0<\varepsilon<\varepsilon(m)$ to be small enough according to the dimension $m$.

\section{HÖLDER CONTINUITY}

Definition 2.1. Let $U \subset \mathbb{R}^{m}$ be open, $B(x, r) \subset U, u: U \rightarrow \mathbb{R}$, and $\varepsilon>0$. We say that $u$ is linearly $\varepsilon$-approximatable at $(x, r)$ if there exists $e \in \mathbb{R}^{m}$ such that

$$
|u(x+h)-u(x)-\langle e, h\rangle| \leqslant \varepsilon r
$$

whenever $h \in B(0, r)$. Such an $e$ is called an $\varepsilon$-approximate gradient of $u$ at $(x, r)$ and we let

$\operatorname{grad}(u, x, r, \varepsilon):=\mathbb{R}^{m} \cap\{e:|u(x+h)-u(x)-\langle e, h\rangle| \leqslant \varepsilon r$ for every $h \in B(0, r)\}$.

In case $u$ is linearly $\varepsilon$-approximatable at $(x, r)$ we also define

$$
|\operatorname{grad}|(u, x, r, \varepsilon):=\sup \{|e|: e \in \operatorname{grad}(u, x, r, \varepsilon)\} .
$$

Furthermore we say that $u$ is linearly approximatable at $x$ if for every $\varepsilon>0$ there exists $r>0$ such that $u$ is linearly $\varepsilon$-approximatable at $(x, r)$.

One observes that $u$ is differentiable at $x$ if and only if $u$ is linearly approximatable at $x$ and one can take $e$ in the definition independently of $\varepsilon$. Therefore the cases of interest arise when $e$ does not approach a limit as $\varepsilon \rightarrow 0$. The following example is due to D. Preiss as reported in [3].

Example 2.2. Let $m=1$ and $u(x)=x \sin (\ln |\ln | x||), x \in \mathbb{R} \backslash\{0\}, u(0)=0$. It is an easy matter to check that $u$ is Lipschitzian when restricted to some neighborhood of 0 . Furthermore, given $h>0$ one sees that

$$
\frac{u(h)-u(0)}{r_{j}}=h \sin \left(\ln \left|\ln r_{j}+\ln h\right|\right)
$$

whenever $r_{j}>0, j=1,2, \ldots$ Given $-1 \leqslant t \leqslant 1$ and choosing properly the sequence $r_{j} \rightarrow 0$ as $j \rightarrow \infty$ one can readily achieve

$$
\sup \left\{\left|\frac{u(h)-u(0)}{r_{j}}-t h\right|: h \in K\right\} \rightarrow 0 \text { as } j \rightarrow \infty
$$


for every compact $K \subset \mathbb{R}$, according to Ascoli's Theorem. In other words, for every $\varepsilon>0$ and every $-1 \leqslant t \leqslant 1$ there exists $r>0$ such that $u$ is linearly $\varepsilon$-approximatable at $(0, r)$ and $t \in \operatorname{grad}(u, 0, r, \varepsilon)$.

Interesting conclusions can be drawn when the condition of linear approximatability is met uniformly in $x$, as we will show in this section. We distinguish between the case when $|\operatorname{grad}|(u, x, r, \varepsilon)$ is bounded as $r$ varies, and the case when it is not.

Theorem 2.3. Assume that $U \subset \mathbb{R}^{m}$ is an open ball, $u: U \rightarrow \mathbb{R}$ and $0<r_{0} \leqslant \infty$. The following conditions are equivalent.

(A) $u$ is Lipschitzian in $U$.

(B) There are $\varepsilon>0$ and $C>0$ such that for every $x \in U$ and $0<r<$ $\min \left\{r_{0}, \operatorname{dist}\left(x, \mathbb{R}^{m} \backslash U\right)\right\}$ the function $u$ is linearly $\varepsilon$-approximatable at $(x, r)$ and $|\operatorname{grad}|(u, x, r, \varepsilon) \leqslant C$.

Proof. Assume that $u$ is Lipschitzian. Let $\varepsilon:=\operatorname{Lip} u$ and $C:=2 \operatorname{Lip} u$. Given $x \in U$ and $r>0$ such that $B(x, r) \subset U$ we notice that $|u(x+h)-u(x)| \leqslant(\operatorname{Lip} u)|h| \leqslant \varepsilon r$ whenever $h \in B(0, r)$. This shows that $u$ is linearly $\varepsilon$-approximatable at $(x, r)$ (letting $e=0$ in the definition). Next if $e \in \mathbb{R}^{m} \backslash\{0\}$ is such that $\mid u(x+h)-$ $u(x)-\langle e, h\rangle \mid \leqslant \varepsilon r$ for every $h \in B(0, r)$, then, letting $h:=r|e|^{-1} e$, one infers that $|e| \leqslant 2 \varepsilon$. This means that $|\operatorname{grad}|(u, x, r, \varepsilon) \leqslant C$ and completes the proof that (A) implies (B).

In order to show that (B) implies (A) we let $x, y \in U$ be such that $r:=|x-y|<r_{0}$ and we assume that $B(x, r) \subset U$. If $e$ is an $\varepsilon$-approximate gradient of $u$ at $(x, r)$, then $|e| \leqslant C$ by our assumption, whence $|u(y)-u(x)| \leqslant|\langle e, y-x\rangle|+\varepsilon r \leqslant(C+$ $\varepsilon)|x-y|$. It therefore follows from the convexity of $U$ that $\operatorname{Lip} u \leqslant C+\varepsilon$.

Having shown that in some cases linearly $\varepsilon$-approximatable functions are Lipschitzian, it is interesting to know whether or not all linearly $\varepsilon$-approximatable functions are Lipschitzian. The following example shows that they are not.

Example 2.4. Let $U=\left(-\frac{1}{2}, \frac{3}{2}\right) \subset \mathbb{R}, S=[0,1] \subset \mathbb{R}, \varepsilon>0$ and $R<\frac{1}{12}$. Take $r_{0}=\frac{1}{2}$ and define $u: U \rightarrow \mathbb{R}$ by

$$
u(x)=x R\left(1-\varepsilon(2 \ln (2))^{-1} \ln (|x|)\right) .
$$

We first observe that the derivative

$$
u^{\prime}(x)=R\left(1-\varepsilon(2 \ln (2))^{-1}(\ln (|x|)+1)\right)
$$

for $x \neq 0$. Since $u^{\prime}(x) \rightarrow \infty$ as $x \rightarrow 0$ it follows that neither $u$ nor $u \uparrow S$ are Lipschitzian.

$u$ is, however, linearly $\varepsilon$-approximatable. This can be checked by selecting the elements of $\operatorname{grad}(u, x, r, \varepsilon)$ to be linear approximations of the function between dyadic points at an appropriate scale. That is, it can be checked that, for $x \in S$ and $r \in\left(0, r_{0}\right]$ the following choices of $e_{x, r} \in \operatorname{grad}(u, x, r, \varepsilon)$ are valid:

For $x=0$ and $r \in\left(0, r_{0}\right], r \in\left(2^{-j-1}, 2^{-j}\right]$ for some $j \in \mathbb{N}$. In this case we can take

$$
e_{x, r}=\frac{u\left(2^{-j}\right)-u(0)}{2^{-j}}=R\left(1+\frac{j \varepsilon}{2}\right) .
$$

For $x=2^{-j}$ and $r \in\left(0,3 \cdot 2^{-j}\right]$ for some $j \in \mathbb{N}$ we can take

$$
e_{x, r}=\frac{u(x)-u(x / 2)}{x / 2}=\frac{u\left(2^{-j}\right)-u\left(2^{-j-1}\right)}{2^{-j-1}}=R-\frac{\varepsilon R(j-1)}{2} .
$$


For $x=2^{-j}$ and $r \in\left(3 \cdot 2^{-j}, r_{0}\right]$ for some $j \in \mathbb{N}, x+r \in\left(2^{-k-1}, 2^{-k}\right]$ for some $k \in \mathbb{N}$ and we can take

$$
e_{x, r}=\frac{u\left(2^{-k}\right)-u\left(2^{-k-1}\right)}{2^{-k-1}}=R-\frac{\varepsilon R(k-1)}{2} .
$$

Finally, in any other case, $x \in\left(2^{-j-1}, 2^{-j}\right]$ for some $j \in \mathbb{N}$ and we can take

$$
e_{x, r}=e_{2^{-j}, r}
$$

We can, though, show that linearly $\varepsilon$-approximatable functions do have some degree of regularity. Indeed, we now show that for any $\varepsilon>0$ and any $0<\alpha<$ 1 , any linearly $\varepsilon$-approximatable function is Hölder continuous with exponent $\alpha$. Initially this result may seem a little unexpected since the Reifenberg Theorem only provides for Hölder continuity with exponent $\alpha$ for sufficiently small $\varepsilon, \varepsilon<\varepsilon(\alpha, m)$. Alternatively, we note that if $u$ is 'shallow', then it is in any case Lipschitz and Hölder, but if it is 'steep', then the linear $\varepsilon$-approximation property says that we are 'vertically' within $\varepsilon$ from an affine plane, which implies that a point on the graph is actually much closer to the affine space than $\varepsilon$, the vertical distance. That is, the analogous Reifenberg approximation property improves as the 'slope' of $u$ increases. With this intuition the result is no longer so unexpected.

Lemma 2.5 (Same scale, different center). Assume that $e_{x, r} \in \operatorname{grad}(u, x, r, \varepsilon)$, $e_{y, r} \in \operatorname{grad}(u, y, r, \varepsilon)$, and $|y-x| \leqslant r / 2$. One then has $\left|e_{y, r}\right| \leqslant\left|e_{x, r}\right|+6 \varepsilon$.

Proof. First notice that

$$
\left\langle e_{x, r}, y-x\right\rangle-\varepsilon r \leqslant u(y)-u(x) \leqslant\left\langle e_{x, r}, y-x\right\rangle+\varepsilon r .
$$

Letting $z=y+\frac{1}{2} r\left|e_{y, r}\right|^{-1} e_{y, r}$, one has $|z-x| \leqslant r$, whence

$$
\left\langle e_{x, r}, x-z\right\rangle-\varepsilon r \leqslant u(x)-u(z) \leqslant\left\langle e_{x, r}, x-z\right\rangle+\varepsilon r .
$$

Summing (2) and (3) yields

$$
|u(y)-u(z)| \leqslant\left|\left\langle e_{x, r}, y-z\right\rangle\right|+2 \varepsilon r \leqslant\left|e_{x, r}\right| \frac{r}{2}+2 \varepsilon r .
$$

Consequently,

$$
\begin{aligned}
\left|e_{y, r}\right| \frac{r}{2} & =\left|\left\langle e_{y, r}, z-y\right\rangle\right| \\
& \leqslant\left|u(z)-u(y)-\left\langle e_{y, r}, z-y\right\rangle\right|+|u(z)-u(y)| \\
& \leqslant \varepsilon r+\left|e_{x, r}\right| \frac{r}{2}+2 \varepsilon r,
\end{aligned}
$$

and in turn,

$$
\left|e_{y, r}\right| \leqslant\left|e_{x, r}\right|+6 \varepsilon
$$

Lemma 2.6 (Same center, different scale). Assume that $e_{x, r} \in \operatorname{grad}(u, x, r, \varepsilon)$, and $e_{x, 2 r} \in \operatorname{grad}(u, x, 2 r, \varepsilon)$. One then has $\left|e_{x, r}\right| \leqslant\left|e_{x, 2 r}\right|+3 \varepsilon$.

Proof. Letting $y=x+r\left|e_{x, r}\right|^{-1} e_{x, r}$, one notices $|y-x| \leqslant r$, whence

$$
|u(y)-u(x)| \leqslant\left|\left\langle e_{x, 2 r}, y-x\right\rangle\right|+2 \varepsilon r .
$$


Therefore

$$
\begin{aligned}
\left|e_{x, r}\right| r & \leqslant\left|\left\langle e_{x, r}, y-x\right\rangle\right| \\
& \leqslant\left|u(y)-u(x)-\left\langle e_{x, r}, y-x\right\rangle\right|+|u(y)-u(x)| \\
& \leqslant \varepsilon r+\left|e_{x, 2 r}\right| r+2 \varepsilon r .
\end{aligned}
$$

Finally

$$
\left|e_{x, r}\right| \leqslant\left|e_{x, 2 r}\right|+3 \varepsilon
$$

In the remaining part of this paper we use the notation $B\left(S, r_{0}\right)$ to denote the $r_{0}$-neighborhood of a set $S \subset \mathbb{R}^{m}$, i.e.

$$
B\left(S, r_{0}\right):=\mathbb{R}^{m} \cap\left\{y: \operatorname{dist}(y, S) \leqslant r_{0}\right\} .
$$

Theorem 2.7. Assume that:

(A) $\varepsilon>0, U \subset \mathbb{R}^{m}$ is open, $u: U \rightarrow \mathbb{R}, S \subset U$ is compact and $r_{0}>0$ is such that $B\left(S, r_{0}\right) \subset U$;

(B) for every $0<r \leqslant r_{0}$ and every $x \in S$ the function $u$ is linearly $\varepsilon$ approximatable at $(x, r)$.

It then follows that $u \in C^{0, \alpha}(S)$ for every $0<\alpha<1$.

Proof. Since $S$ is compact we can cover $S$ by finitely many balls of radius $r_{0} / 2$, $B\left(x_{i}, r_{0} / 2\right), i=1, \ldots, Q$. We choose $e_{x, r} \in \operatorname{grad}(u, x, r, \varepsilon)$ for every $x \in S$ and $0<r \leqslant r_{0}$. Letting

$$
E:=\max \left\{\left|e_{x_{i}, r_{0}}\right|: i=1, \ldots, Q\right\}<\infty
$$

we infer from Lemma 2.5 that, for every $x \in S$,

$$
\left|e_{x, r_{0}}\right| \leqslant E+6 \varepsilon .
$$

Applying inductively Lemma 2.6 we obtain

$$
\left|e_{x, 2^{-k} r_{0}}\right| \leqslant E+6 \varepsilon+3 k \varepsilon
$$

whenever $k \in \mathbb{N}$. If $y \in B\left(x, 2^{-k} r_{0}\right)$, then

$$
\begin{aligned}
|u(y)-u(x)| & \leqslant\left|\left\langle e_{x, 2^{-k}} r_{0}, y-x\right\rangle\right|+\varepsilon 2^{-k} r_{0} \\
& \leqslant\left|e_{x, 2^{-k} r_{0}}\right| 2^{-k} r_{0}+\varepsilon 2^{-k} r_{0} \\
& \leqslant 2^{-k} r_{0}(E+7 \varepsilon+3 k \varepsilon) .
\end{aligned}
$$

Now, take any $0<\alpha<1$. In the case that $|y-x|=2^{-k} r_{0}$ for some $k \in \mathbb{N}$ we thus have

$$
\frac{|u(y)-u(x)|}{|y-x|^{\alpha}} \leqslant r_{0}^{1-\alpha}\left(2^{\alpha-1}\right)^{k}(E+7 \varepsilon+3 k \varepsilon) .
$$

Since the above sequence in $k$ converges to 0 as $k \rightarrow \infty$ there exists $C \in \mathbb{R}$ (depending only upon $\alpha, r_{0}, E$, and $\varepsilon$ ) such that for each $k \in \mathbb{N}, x \in S$, and $y \in U$ satisfying $|y-x|=2^{-k} r_{0}$,

$$
|u(y)-u(x)| \leqslant C|y-x|^{\alpha} .
$$

Now consider $y \in S \cap B\left(x, r_{0}\right)$. Then there exists $k \in \mathbb{N}$ such that $2^{-k-1} r_{0} \leqslant$ $|y-x|<2^{-k} r_{0}$. It follows from (44) that

$$
\frac{|u(y)-u(x)|}{|y-x|^{\alpha}} \leqslant 2^{\alpha} r_{0}^{1-\alpha}\left(2^{\alpha-1}\right)^{k}(E+7 \varepsilon+3 k \varepsilon) \leqslant 2^{\alpha} C .
$$


That is,

$$
|u(y)-u(x)| \leqslant 2^{\alpha} C|y-x|^{\alpha} .
$$

Moreover, letting

$$
F:=\max \left\{\left|u\left(x_{i}\right)\right|: i=1, \ldots, Q\right\},
$$

we notice that, since $S \subset \bigcup_{i=1}^{Q} B\left(x_{i}, r_{0}\right)$,

$$
|u(x)| \leqslant F+E r_{0}+\varepsilon r_{0}=: M
$$

for each $x \in S$. We thus deduce that for $|y-x| \geqslant r_{0}$,

$$
|u(y)-u(x)| \leqslant r_{0}^{-\alpha} 2 M|y-x|^{\alpha} .
$$

It follows from inequalities (5) and (6) that

$$
|u(y)-u(x)| \leqslant C^{\prime}|y-x|^{\alpha}
$$

for all $y, z \in S$, where $C^{\prime}=\max \left\{2^{\alpha} C, r_{0}^{-\alpha} 2 M\right\}$.

\section{GENERICITY}

Theorem 2.7 implies that the appropriate space of functions in which to consider the genericity of linearly approximatable functions is that of functions Hölder continuous for each $0<\alpha<1$, that is,

$$
\operatorname{Höld}(U):=\bigcap\left\{C^{0, \alpha}(U): 0<\alpha<1\right\} .
$$

We recall that $C^{0, \alpha}(U)$ (the collection of bounded Hölder continuous functions with exponent $\alpha)$ is a Banach space under the norm $\|u\|_{\alpha}:=\|u\|_{\infty}+\operatorname{Höld}_{\alpha}(u)$, where $\|u\|_{\infty}:=\sup \{|u(x)|: x \in U\}$ and

$$
\operatorname{Höld}_{\alpha}(u):=\sup \left\{\frac{|u(y)-u(x)|}{|y-x|^{\alpha}}: x, y \in U \text { and } x \neq y\right\} .
$$

To make Höld $(U)$ a metric space we need to consider simultaneously all appropriately large $0<\alpha<1$. We therefore take $\alpha_{j}=1-\frac{1}{j+1}$ for each $j=1,2, \ldots$ and define for $u, v \in \operatorname{Höld}(U)$,

$$
d(u, v):=\sum_{j=1}^{\infty} \frac{1}{2^{j}}\left(\frac{\|u-v\| \|_{\alpha_{j}}}{1+\|u-v\|_{\alpha_{j}}}\right) .
$$

It is clear that $\operatorname{Höld}(U)$ is complete under the given metric.

We show that the collection of functions linearly $\varepsilon$-approximatable at at least one point is meager in $\operatorname{Höld}(U)$. To make this statement more rigorous we make the following definition.

Definition 3.1. Let $U \subset \mathbb{R}^{m}$ be open, $S \subset U$ be compact, $r_{0}>0$ such that $B\left(S, r_{0}\right) \subset U$, and $\varepsilon>0$. We write $L A\left(\varepsilon, r_{0}, S, U\right)$ to denote the set of those functions $u \in \operatorname{Höld}(U)$ with the following property. There exists $x \in S$ such that for every $0<r \leqslant r_{0}, u$ is linearly $\varepsilon$-approximatable at $(x, r)$.

Lemma 3.2. If $u_{j} \in L A\left(\varepsilon, r_{0}, S, U\right), j=1,2, \ldots$, converge uniformly to $u \in$ $\operatorname{Höld}(U)$, then $u \in L A\left(\varepsilon, r_{0}, S, U\right)$. 
Proof. For each $j=1,2, \ldots$, we select $x_{j} \in S$ such that $u_{j}$ is linearly $\varepsilon$-approximatable at $\left(x_{j}, r\right)$ whenever $0<r \leqslant r_{0}$. Passing to a subsequence if necessary we will assume that $x_{j} \rightarrow x \in S$ as $j \rightarrow \infty$. From now on we fix an $0<r \leqslant r_{0}$ and we select and fix an $e_{j}\left(x_{j}, r\right) \in \operatorname{grad}\left(u_{j}, x_{j}, r, \varepsilon\right)$. From the convergence property of $u_{1}, u_{2}, \ldots$ and the Arzelà-Ascoli Theorem we infer that there exists $\delta>0$ such that $\left|u_{j}(y)-u_{j}(z)\right| \leqslant 1$ for every $j=1,2, \ldots$ and every $y, z \in B\left(S, r_{0}\right)$ with $|y-z| \leqslant \delta$. Further, there is an integer $j_{0}$ such that: (i) $\left|u_{j}(y)-u_{j_{0}}(y)\right| \leqslant 1$ for each $j \geqslant j_{0}$ and for each $y \in U$, and (ii) $\left|x_{j_{0}}-x_{j}\right| \leqslant \delta$ for every $j \geqslant j_{0}$. We deduce that for any $j \geqslant j_{0}$ and $h \in B(0, r)$,

$$
\begin{aligned}
\left|\left\langle e_{j_{0}}\left(x_{j_{0}}, r\right)-e_{j}\left(x_{j}, r\right), h\right\rangle\right| \leqslant & \left|\left\langle e_{j_{0}}\left(x_{j_{0}}, r\right), h\right\rangle+u_{j_{0}}\left(x_{j_{0}}\right)-u_{j_{0}}\left(x_{j_{0}}+h\right)\right| \\
& +\left|\left\langle e_{j}\left(x_{j}, r\right), h\right\rangle-u_{j}\left(x_{j}\right)+u_{j}\left(x_{j}+h\right)\right| \\
& +\left|u_{j_{0}}\left(x_{j_{0}}+h\right)-u_{j}\left(x_{j_{0}}+h\right)\right| \\
& +\left|u_{j}\left(x_{j_{0}}+h\right)-u_{j}\left(x_{j}+h\right)\right| \\
& +\left|u_{j}\left(x_{j}\right)-u_{j}\left(x_{j_{0}}\right)\right| \\
& +\left|u_{j}\left(x_{j_{0}}\right)-u_{j_{0}}\left(x_{j_{0}}\right)\right| \\
\leqslant & 2 \varepsilon r+4 .
\end{aligned}
$$

It follows that $e_{1}\left(x_{1}, r\right), e_{2}\left(x_{2}, r\right), \ldots$ is a bounded sequence in $\mathbb{R}^{m}$ and thus that a convergent subsequence, still denoted $e_{1}\left(x_{1}, r\right), e_{2}\left(x_{2}, r\right), \ldots$, exists with limit $e(x, r) \in \mathbb{R}^{m}$. Now let $\eta>0$ and choose $\delta>0$ such that $\left|u_{j}(y)-u_{j}(z)\right| \leqslant \eta$ for every $j=1,2, \ldots$ and $y, z \in B\left(S, r_{0}\right)$ with $|y-z| \leqslant \delta$. Next take $k$ such that $\left|u_{k}(y)-u(y)\right| \leqslant \eta$ for all $y \in U$ and such that $\max \left\{\left|x_{k}-x\right|,\left|e_{k}\left(x_{k}, r\right)-e(x, r)\right|\right\} \leqslant$ $\min \{\eta, \delta\}$. Then, for all $h \in B(0, r)$,

$$
\begin{aligned}
|u(x+h)-u(x)-\langle e(x, r), h\rangle| \leqslant & \left|u_{k}\left(x_{k}+h\right)-u_{k}\left(x_{k}\right)-\left\langle e_{k}\left(x_{k}, r\right), h\right\rangle\right| \\
& +\left|u(x+h)-u_{k}(x+h)\right| \\
& +\left|u_{k}(x+h)-u_{k}\left(x_{k}+h\right)\right| \\
& +\left|u_{k}\left(x_{k}\right)-u\left(x_{k}\right)\right| \\
& +\left|u\left(x_{k}\right)-u(x)\right| \\
& +\left|\left\langle e_{k}\left(x_{k}, r\right)-e(x, r), h\right\rangle\right| \\
\leqslant & \varepsilon r+\eta(4+|h|) .
\end{aligned}
$$

Since this is true for all $\eta>0, u$ is linearly $\varepsilon$-approximatable at $(x, r)$. The conclusion follows from the arbitrariness of $r$.

Theorem 3.3. Let $U \subset \mathbb{R}^{m}$ be open and let $\mathscr{I}$ denote the collection of those functions $u \in \operatorname{Höld}(U)$ with the property that for each $x \in U$ and each $\varepsilon>0$ there are $r_{j} \downarrow 0$ as $j \uparrow \infty$ such that $u$ is not linearly $\varepsilon$-approximatable at $\left(x, r_{j}\right)$. Then $\mathscr{I}$ is residual in $\operatorname{Höld}(U)$.

Proof. For each $k=1,2, \ldots$ we define $S_{k}:=U \cap B(0, k) \cap\left\{x: \operatorname{dist}\left(x, \mathbb{R}^{m} \backslash U\right) \geqslant\right.$ $\left.k^{-1}\right\}$. We observe that

$$
\mathscr{I}=\operatorname{Höld}(U) \backslash\left(\bigcup_{k=1}^{\infty} \bigcup_{j=1}^{\infty} \bigcup_{i=1}^{\infty} L A\left(j, \frac{1}{2 k i}, S_{k}, U\right)\right) .
$$

According to the Baire Category Theorem, it therefore suffices to show that each $L A\left(\varepsilon, r_{0}, S, U\right)$ is meager in $\operatorname{Höld}(U)$. 
We first observe that $L A\left(\varepsilon, r_{0}, S, U\right)$ is closed in $\operatorname{Höld}(U)$, as readily follows from Lemma 3.2. We now need to show that $\operatorname{Höld}(U) \backslash L A\left(\varepsilon, r_{0}, S, U\right)$ is dense in $\operatorname{Höld}(U)$. Let $u \in \operatorname{Höld}(U)$ and $\eta>0$ be fixed for the remaining part of this proof. We need to find $u^{*} \in \operatorname{Höld}(U)$ such that $d\left(u, u^{*}\right) \leqslant \eta$ and $u^{*} \notin L A\left(\varepsilon, r_{0}, S, U\right)$.

We see first that there exists an integer $j_{0} \in \mathbb{N}$ such that for any $v \in \operatorname{Höld}(U)$,

$$
\sum_{j=j_{0}+1}^{\infty} \frac{1}{2^{j}}\left(\frac{\|v\| \|_{\alpha_{j}}}{1+\|v\| \|_{\alpha_{j}}}\right)<\frac{\eta}{4}
$$

Put $\alpha:=\alpha_{j_{0}}+\frac{1}{2\left(j_{0}+1\right)}=1-\frac{1}{2\left(j_{0}+1\right)}$ and notice that $\alpha<1$. Since $u \in C^{0, \alpha}(U)$ it admits an extension $\hat{u} \in C^{0, \alpha}\left(\mathbb{R}^{m}\right)$; see $\left[\underline{6}\right.$. Given $\delta>0$ we let $\Phi_{\delta}$ denote a smooth regularization kernel such that $\operatorname{spt} \Phi_{\delta} \subset B(0, \delta)$. We notice that for every $y, z \in \mathbb{R}^{m}$,

$$
\begin{aligned}
\mid\left(\Phi_{\delta} * \hat{u}-\hat{u}\right)(y) & -\left(\Phi_{\delta} * \hat{u}-\hat{u}\right)(x) \mid \\
& \leqslant \int_{\mathbb{R}^{m}} \Phi_{\delta}(z)|\hat{u}(y-z)-\hat{u}(y)-\hat{u}(x-z)+\hat{u}(x)| d z \\
& \leqslant 2 \operatorname{Höld}_{\alpha}(\hat{u}) \int_{B(0, \delta)} \Phi_{\delta}(z) \min \left\{|z|^{\alpha},|y-x|^{\alpha}\right\} d z \\
& \leqslant \delta^{\frac{1}{2\left(j_{0}+1\right)}} 2 \operatorname{Höld}_{\alpha}(\hat{u})|y-x|^{\alpha_{j_{0}}} .
\end{aligned}
$$

(The last inequality follows after distinguishing between the cases when $|y-x| \leqslant \delta$ and when $|z| \leqslant \delta \leqslant|y-x|$.) It readily follows that there exists $\delta>0$ small enough for $\| u-\left(\Phi_{\delta} * \hat{u}\right)\left\lceil U \|_{\alpha_{j}}<\eta / 4\right.$ whenever $j=1, \ldots, j_{0}$. Letting $u^{\prime}:=\left(\Phi_{\delta} * \hat{u}\right)\lceil U$ we infer that

$$
d\left(u, u^{\prime}\right)=\sum_{j=1}^{j_{0}} \frac{1}{2^{j}}\left(\frac{\left\|u-u^{\prime}\right\|_{\alpha_{j}}}{1+\mid\left\|u-u^{\prime}\right\|_{\alpha_{j}}}\right)+\sum_{j=j_{0}+1}^{\infty} \frac{1}{2^{j}}\left(\frac{\left\|u-u^{\prime}\right\|_{\alpha_{j}}}{1+\left\|u-u^{\prime}\right\| \|_{\alpha_{j}}}\right)<\frac{\eta}{2} .
$$

Observe also that $u^{\prime} \in \operatorname{Lip}(U) \subset \operatorname{Höld}(U)$ and define $\lambda:=\operatorname{Lip} u^{\prime}$.

We now define $\mu:=4(1+\lambda+\varepsilon)$, and corresponding to each $0<r \leqslant r_{0}$ we define $u_{\mu, r}: \mathbb{R}^{m} \rightarrow \mathbb{R}$ by $u_{\mu, r}(x):=\mu \operatorname{dist}\left(x, r \mathbb{Z} \times \mathbb{R}^{m-1}\right)$. We note that $\left\|u_{\mu, r}\right\|_{\infty} \leqslant \mu r / 2$ and that $\operatorname{Höld}_{\gamma}\left(u_{\mu, r}\right) \leqslant \mu r^{1-\gamma}, 0<\gamma<1$ (in order to establish the latter notice that $\operatorname{Lip} u_{\mu, r}=\mu$ and for every $y, z \in \mathbb{R}^{m}$ either $|y-z| \leqslant r$ and $\left|u_{\mu, r}(y)-u_{\mu, r}(z)\right| \leqslant$ $\mu r^{1-\gamma}|y-z|^{\gamma}$ or $|y-z|>r$ and there exists $z^{\prime} \in \mathbb{R}^{m}$ with $\left|y-z^{\prime}\right| \leqslant r$ and $\left.u_{\mu, r}\left(z^{\prime}\right)=u_{\mu, r}(z)\right)$. Therefore there exists an $0<r \leqslant r_{0}$ sufficiently small for $\left\|u_{\mu, r}\right\|_{\alpha_{j}}<\eta / 4$ whenever $j=1, \ldots, j_{0}$. Letting $u^{*}:=u^{\prime}+u_{\mu, r}$ we deduce as above that $d\left(u^{\prime}, u^{*}\right)<\eta / 2$, and in turn $d\left(u, u^{*}\right)<\eta$.

Assume if possible that $u^{*} \in L A\left(\varepsilon, r_{0}, S, U\right)$. There would exist $x \in S$ such that $u^{*}$ is linearly $\varepsilon$-approximatable at $(x, r)$. From the definition of $u_{\mu, r}$ we infer that there are $r / 4 \leqslant t^{ \pm} \leqslant r$ such that either

or

$$
\min \left\{u\left(x+t^{+} e_{1}\right)-u(x), u\left(x-t^{-} e_{1}\right)-u(x)\right\} \geqslant \frac{\mu r}{4}
$$

$$
\max \left\{u\left(x+t^{+} e_{1}\right)-u(x), u\left(x-t^{-} e_{1}\right)-u(x)\right\} \leqslant-\frac{\mu r}{4} .
$$

Pick $e \in \operatorname{grad}\left(u^{*}, x, r, \varepsilon\right)$. Using the vectors $h^{ \pm}:= \pm t^{ \pm} e_{1}$ in the definition of linear approximatability we find, in the first case, that

$$
\left\langle e, t^{+} e_{1}\right\rangle \geqslant-\lambda r+\frac{\mu r}{4}-\varepsilon r=r
$$


and

$$
-\left\langle e, t^{-} e_{1}\right\rangle \geqslant-\lambda r+\frac{\mu r}{4}-\varepsilon r=r
$$

a contradiction. The second case yields to the analogous contradiction.

\section{LEVEL SETS}

Theorem 4.1. There exists $\varepsilon(m)>0$ with the following property. Assume that

(A) $U \subset \mathbb{R}^{m}$ is open, $u: U \rightarrow \mathbb{R}, B\left(x_{0}, r_{0}\right) \subset U$, and $\lambda_{0}>0$;

(B) $0<\varepsilon \leqslant \varepsilon(m) \lambda_{0}$;

(C) for every $0<r \leqslant r_{0}$ and every $x \in B\left(x_{0}, r_{0}\right)$ the function $u$ is linearly $\varepsilon$-approximatable at $(x, r)$;

(D) for every $0<r \leqslant r_{0}$ and every $x \in B\left(x_{0}, r_{0}\right)$ one has $|\operatorname{grad}|(u, x, r, \varepsilon) \geqslant \lambda_{0}$. It follows that a neighborhood of $x_{0}$ in the level set $U \cap\left\{x: u(x)=u\left(x_{0}\right)\right\}$ is bi-Hölder homeomorphic to an $(m-1)$-dimensional ball.

Proof. Put $\Sigma=U \cap\left\{x: u(x)=u\left(x_{0}\right)\right\}$. Given $x \in \Sigma \cap B\left(x_{0}, r_{0}\right)$ and $0<r \leqslant r_{0}$ we choose $e_{x, r} \in \operatorname{grad}(u, x, r, \varepsilon)$ with $\left|e_{x, r}\right| \geqslant \lambda_{0}$. Notice that

$$
\left|\left\langle e_{x, r}, y-x\right\rangle\right|=\left|u(y)-u(x)-\left\langle e_{x, r}, y-x\right\rangle\right| \leqslant \varepsilon r
$$

whenever $y \in \Sigma \cap B(x, r)$. In other words,

$$
y \in B\left(x+W_{x, r}, \lambda_{0}^{-1} \varepsilon r\right)
$$

where $W_{x, r}:=\mathbb{R}^{m} \cap\left\{h:\left\langle h, e_{x, r}\right\rangle=0\right\}$. The other way around, assume $h_{0} \in$ $W_{x, r} \cap B(x, r / 2)$. Letting $h_{t}:=h_{0}+t e_{x, r}\left|e_{x, r}\right|^{-1}$ we infer that

$$
u\left(x+h_{t}\right) \leqslant u(x)+\left\langle e_{x, r}, h_{t}\right\rangle+\varepsilon r=u(x)+t\left|e_{x, r}\right|+\varepsilon r
$$

and that

$$
u\left(x+h_{t}\right) \geqslant u(x)-\left\langle e_{x, r}, h_{t}\right\rangle-\varepsilon r \geqslant u(x)-t\left|e_{x, r}\right|-\varepsilon r
$$

(notice that $\left|h_{t}\right| \leqslant r$ provided $t^{2} \leqslant 3 r^{2} / 4$ ). In particular (if $\lambda_{0}^{-1} \varepsilon$ is small enough) $u\left(x+h_{-2\left|e_{x, r}\right|^{-1} \varepsilon r}\right)<u(x)$ and $u\left(x+h_{2\left|e_{x, r}\right|^{-1} \varepsilon r}\right)>u(x)$. Since $t \mapsto h\left(x+h_{t}\right)$ is continuous (according to Theorem 2.7) we see that there exists $-2 \lambda_{0}^{-1} \varepsilon r \leqslant t^{*} \leqslant$ $2 \lambda_{0}^{-1} \varepsilon r$ such that $u\left(x+h_{t^{*}}\right)=u(x)=u\left(x_{0}\right)$, i.e. $x+h_{t^{*}} \in \Sigma$. In other words,

$$
x+h_{0} \in B\left(\Sigma, 2 \lambda_{0}^{-1} \varepsilon r\right) .
$$

The conclusion now follows from Reifenberg's Topological Disk Theorem; see e.g. [9, 8, 11, 4].

\section{REFERENCES}

1. G. Aronsson, M.G. Crandall, and P. Juutinen, A tour of the theory of absolutely minimizing functions, Bull. Amer. Math. Soc. 41 (2004), no. 4, 439-505. MR2083637 (2005k:35159)

2. S. Banach, Über die bairesche kategorie gewisser funktionenmengen, Stud. Math. 3 (1931), $174-179$.

3. M.G. Crandall and L.C. Evans, A remark on infinity harmonic functions, Electron. J. Diff. Eqns. Conf. 06 (2001), 123-129. MR.1804769 (2001j:35076)

4. G. David, Th. De Pauw, and T. Toro, Extensions of Reifenberg's topological disk theorem, Geom. Funct. Anal. (to appear).

5. Th. De Pauw and P. Huovinen, Points of $\varepsilon$-differentiability of Lipschitz functions from $\mathbb{R}^{n}$ to $\mathbb{R}^{n-1}$, Bull. London Math. Soc. 34 (2002), 539-550. MR1912876 (2003f:26014)

6. F. Grünbaum and E.H. Zarantonello, On the extension of uniformly continuous mappings, Michigan Math. J. 15 (1968), 65-74. MR0223506 (36:6554)

7. S. Mazurkiewicz, Sur les fonctions non dérivables, Stud. Math 3 (1931), 92-94. 
8. C.B. Morrey Jr., Multiple integrals in the calculus of variations, Springer, 1966. MR0202511 $(34: 2380)$

9. E.R. Reifenberg, Solution of the Plateau problem for $m$-dimensional surfaces of varying topological type, Acta Math. 104 (1960), 1-92. MR0114145 (22:4972)

10. O. Savin, $C^{1}$ regularity for infinity harmonic functions in two dimensions, Arch. Ration. Mech. Anal. 176 (2005), 351-361. MR2185662 (2006i:35108)

11. T. Toro, Geometric conditions and existence of bi-Lipschitz parameterizations, Duke Math. J. 77 (1995), no. 1, 193-227. MR.1317632 (96b:28006)

Université Catholique de Louvain, Département de Mathématiques, Chemin du Cyclotron, 2, B-1348 Louvain-la-Neuve, Belgique

E-mail address: thierry.depauw@uclouvain.be

Mathematisches Institut der Universität Tübingen, Auf der Morgenstelle 10, 72076 Tübingen, Germany

E-mail address: akoeller@everest.mathematik.uni-tuebingen.de 